\title{
PHƯƠNG THỨC GIAO TIẾP VỚI ĐÔC GIẢ CỦA CÁC BÀI BÌNH LUẬn BÁO CHÍ VỀ "HỔ SƠ PANAMA" TỪ GÓC NHÌN CỦA THUYẾT ĐÁNH GIÁ
}

\author{
Nguyễn Thị Thu Hiền*
}

\author{
Khoa Ngoại ngũu, Đại học Quy Nhơn, 170 An Dưong Vuoong, \\ TP. Quy Nhơn, Bình Định, Việt Nam \\ Nhận bài ngày 17 tháng 05 năm 2016
}

Chỉnh sửa ngày 16 tháng 12 năm 2016; Chấp nhận đăng ngày 06 tháng 01 năm 2017

Tóm tắt: Việc sử dụng các nguồn ngôn liệu của Thuyết đánh giá để phân tích các nét nghĩa liên nhân của một diễn ngôn đang ngày càng trở nên phổ biến, đặc biệt là của các diễn ngôn báo chí. Bài viết này chọn nguồn tham thoại để nghiên cứu vị trí người viết bình luận tin chọn cho mình và cách thức họ tương tác với độc giả dựa trên 30 bài viết về "Hồ sơ Panama". Kết quả nghiên cứu cho thấy đa nguồn xuất hiện nhiều hơn đơn nguồn và đa nguồn mở rộng được ưu tiên hơn đa nguồn hạn định trong thể loại này. Kết luận này cho thấy mức độ giao tiếp cao giữa người viết và độc giả. Với hai yếu tố đa nguồn hạn định và đa nguồn mở rộng, người bình tin đã tạo sự an toàn bằng việc sử dụng các nguồn ngôn liệu đánh giá từ các nguồn khác nhiều hơn từ chính bản thân, hoặc tạo cơ hội cho người đọc tự đánh giá sự kiện.

Tù khóa: Thuyết đánh giá, tham thoại, đa nguồn hạn định, đa nguồn mở rộng, đơn nguồn

\section{Phần mở đầu}

Diễn ngôn bình luận báo chí được cho là một thể loại có tính chủ quan cao của người viết vì nó thường là diễn ngôn thể hiện quan điểm, hay chính kiến về một sự kiện nào đó. Người viết bình luận có nhiều cách để nêu đánh giá và tạo ảnh hưởng lên người đọc tùy thuộc vào mức độ hiểu thông tin của mình. Nếu chọn đánh giá sự việc một cách hiển ngôn, người viết đã nhận trách nhiệm về các thông tin và ý kiến được nêu ra, ngược lại, nếu trình bày quan điểm một cách hàm ẩn thì người viết có thể tách mình ra khỏi các trách nhiệm đối với thông tin được đánh giá. Mặc dù khác về cách bày tỏ chính kiến, nhưng ở một chừng mực nào đó, hiển ngôn hay hàm ẩn đều giúp tạo sự tương tác giữa người bình tin và người đọc tin.

Mục tiêu của bài báo này là nghiên cứu các phương thức giao tiếp với độc giả của

* ĐT.: 84-983443901, Email: nguyenthithuhien@qnu.edu.vn người bình tin thông qua cách chọn nguồn thể hiện quan điểm của người viết. Để đạt được mục tiêu này, chúng tôi sử dụng các nguồn tham thoại (Engagement) trong khung lý thuyết Đánh giá (Appraisal Theory) của Martin và White (2005) vì theo hai tác giả này, tham thoại cho phép nhà nghiên cứu hiểu được cách thức người viết thể hiện sự hiện diện của mình trong văn bản, cách thức tương tác với người đọc và cách họ kiến tạo suy nghĩ của người đọc. Mặc dù khung phân tích này được phát triển dựa trên ngôn ngữ Anh, nhưng các nhà nghiên cứu Vo (2011), và Tran (2011) đã cho rằng thuyết này dựa trên các cấp độ về ngữ nghĩa và từ vựng - ngữ pháp nên có thể áp dụng như một công cụ phân tích tiếng Việt khá thành công.

Ngữ liệu sử dụng để phân tích là 30 bài bình luận về vấn đề thời sự thế giới - "Hồ sơ Panama" được đăng trên trang Vnexpress và Tuổi trẻ online từ ngày 5/4/ 2016 đến ngày 
$10 / 5 / 2016$. Đơn vị phân tích của bài báo là 895 câu trong khối ngữ liệu và được mã hóa từ $\mathrm{C} 1$ đến $\mathrm{Cn}$ theo trật tự của câu xuất hiện trong bài báo. Các văn bản bình luận được mã hóa từ $\mathrm{V} 1$ cho đến V30. Vì vậy, C32V1 có nghĩa là câu thứ 32 của văn bản bình luận 1 .

\section{Thuyết đánh giá}

Phát triển từ ngữ pháp chức năng của Halliday (1994), thuyết đánh giá nghiên cứu hệ thống nghĩa liên nhân của văn bản thông qua ba nguồn: thái độ (Attitude), phân tầng (Graduation) và tham thoại (Engagement) (White, 2003) (thuật ngũ̃ được dịch theo Nguyen, 2016)

Thái độ bao gồm ba bình diện: cảm xúc (Affect), phán xét (Judgement) và đánh giá cảm quan (Appreciation). Trong đó, cảm xúc liên quan đến các yếu tố ngôn ngữ diễn đạt cảm xúc tích cực hay tiêu cực của một con người về một sự kiện được trình bày trong diễn ngôn. Phán xét diễn tả sự đánh giá của người ta về hành vi của con người trong sự kiện, và đánh giá cảm quan bàn đến việc đánh giá các sự kiện hay đồ vật chung quanh sự kiện đó.

Phân tầng chỉ ra các mức độ cao thấp của cảm xúc hay thái độ của con người đối với các yếu tố liên quan đến sự kiện.

Tham thoại bàn đến các nguồn ngôn liệu giúp tác giả của một diễn ngôn thể hiện quan điểm của mình thông qua các nguồn khác nhau. Có hai tiểu hệ thống trong tham thoại: Đơn nguồn (Monogloss) và Đa nguồn (Heterogloss). Đơn nguồn là một dạng diễn ngôn người viết trình bày về một sự thật, không kèm theo bất kỳ yếu tố đánh giá nào. Ví dụ, diễn ngôn C23V2 là một đơn nguồn vì nó chỉ là một mô tả về thực tế cách thức hoạt động của một cơ quan.

C23V2: Nguời đúng đầu USAID điều hành hoạt động của co quan này theo các chỉ thị của Bộ Ngoại giao Mỹ.

Ngược lại, đa nguồn có chứa các yếu tố tình thái như khẳng định hay phủ định, chắc chắn hay dự đoán, tin tưởng hay nghi ngờ... và quan trọng là chính những nguồn ngôn liệu này sẽ không chỉ giúp người viết thể hiện sự đánh giá của mình mà chúng còn giúp người viết tương tác với các độc giả của họ bằng các ngôn từ diễn đạt sự công nhận, thỏa hiệp, hay mời gọi những thái độ, quan điểm khác (White ,2003). Từ quan điểm này, có thể thấy nếu người viết chọn một quan điểm và ý thức được sự tồn tại của các quan điểm khác về cùng một vấn đề thì họ luôn đặt mình vào những thỏa hiệp tiềm năng với những người đọc được cho là có chung hoặc khác về quan điểm tư tưởng. Ví dụ tại câu $\mathrm{C} 5 \mathrm{~V} 3$, bằng việc trích dẫn từ nguồn tin của người phát ngôn Bộ Ngoại giao Mỹ và cách dùng từ trích dẫn "khẳng định", người bình tin đã hàm ý thể hiện được mức độ chắc chắn của mình về việc "Washington không hề biết trước về quá trình...", và họ muốn người đọc chia sẻ quan điểm này. Đây là loại đa nguồn hạn định (Heterogloss- Contraction).

C5V3: Người phát ngôn Bộ Ngoại giao Mỹ cuñg khẳng định, Washington không hề biết truớc về quá trình cũng nhu kết quả cuộc điều tra vì ho không can thiệp vào công việc của các nhà báo.

Nhưng cách thức thể hiện quan điểm lại hoàn toàn khác trong $\mathrm{C} 17 \mathrm{~V} 3$

C17V3: Ông không nêu tên nhũng nhân vật đã làm điều này, song rất có thể đó là các nhân vật trong Nhà Trắng. 
Bằng việc sử dụng "rất có thể”, tác giả bài báo hàm ý đây chỉ là suy đoán của mình, và độ chắc chắn ở mức độ thấp nên người đọc có quyền tin hoặc không tin vào suy đoán này và được khuyến khích mời gọi bày tỏ quan điểm riêng về vụ việc. Tham thoại này được gọi là đa nguồn mở rộng (Heterogloss - Expansion)

Cho dù sử dụng đa nguồn hạn định hay đa nguồn mở rộng thì rõ ràng người viết đã tạo ra một giao tiếp với những người đọc tiềm năng của mình với các yếu tố tham thoại trên. Tầm quan trọng của tham thoại trong việc giúp người viết tạo vị thế và giao tiếp với người đọc cũng đã được nhiều nhà nghiên cứu ngôn ngữ quan tâm. Vo (2011:166) khi nghiên cứu về các điểm tương đồng và khác biệt trong các bản tin thương mại Anh - Việt đã cho rằng bản tin tiếng Việt có xu hướng sử dụng đơn nguồn hơn là đa nguồn, còn bản tin tiếng Anh lại có xu hướng giao tiếp với độc giả nhiều hơn bởi các yếu tố đa nguồn nhằm gắn thông tin với các nguồn bên ngoài. Allison \& Wu (2005) nghiên cứu các yếu tố đánh giá trong các bài văn tranh luận của sinh viên đã kết luận rằng việc sử dụng đa nguồn mở rộng nhằm giảm đi yếu tố chủ quan của luận điểm thường xảy ra ở các bài văn đạt điểm cao, nhưng đa nguồn hạn định lại thường xuyên xuất hiện trong các bài văn điểm thấp vì chúng làm giảm đi tính tranh luận của luận điểm. Hyland (2005) khi phân tích các yếu tố tham thoại trong các diễn ngôn học thuật đã kết luận rằng tham thoại là một nhân tố quan trọng làm cho độc giả và người viết thành các tham thể của một hội thoại đang được mở ra.

\section{Kết quả phân tích}

\section{1. Đơn nguồn và đa nguồn}

Trong tổng số 895 câu của toàn khối ngữ liệu, có 742 đa nguồn, 153 đơn nguồn. Tỉ lệ này cho thấy bình luận tin là một thể loại diễn ngôn thể hiện quan điểm và có yếu tố tương tác với người đọc cao bằng cách bày tỏ quan điểm từ các nguồn khác nhau.

Câu C12V5 là một đơn nguồn, đơn thuần trình bày khách quan một sự kiện xảy ra. Các hình thức đơn nguồn kiểu này thường xuất hiện ở dạng tường thuật tin hơn là bình luận tin. Khi sử dụng đơn nguồn, người viết hàm ý xác nhận tính tin cậy của thông tin với tư cách là một sự thật.

C12V5: Giới điều tra chú ý đến Mossack Fonseca sau khi phát hiện một loạt căn hộ do ngườ thân của một chính trị gia bị giam giư đưng tên. (đơn nguồn)

Khác với C12V5, C16V26 lại là một điển hình của đa nguồn thông qua hai yếu tố

C16V26: Mossack Fonseca cũng nói việc thành lập các công ty để che giấu danh tính của nhũng người chủ thưc sụ hoàn toàn "không được ủng hộ và là hành động sai trái”. Tuy nhiên, dĩ liệu bị rò rỉ cho thấy, công ty Mossack Fonseca đã lập mạng luới khổng lồ để hỗ trọ hàng loạt chính trị gia và doanh nhân các nuoóc che giấu tài sản và rủa hàng tỷ USD tiền mặt.

Thứ nhất, bằng cách đưa nguồn trích dẫn "Mossack Fonseca cũng nói”, người viết báo muốn độc giả tin rằng thông tin kèm theo không phải là sự thực mà chỉ là phát ngôn của một nhân vật, và vì thế khuyến khích người đọc tự đánh giá về thông tin này. Thứ hai, người viết mở đầu bằng một sự đánh giá tích cực khi người đứng đầu công ty Mossack Fonseca nhận thức về hành động sai trái và kết thúc bằng một sự đánh giá tiêu cực là công ty này đã thực sự làm điều mà họ biết là sai đó. Cách dùng từ "tuy nhiên" để kết nối hai 
câu trên đã cho phép người viết bình luận tự tạo cho mình một cơ hội định hướng suy nghĩ, đánh giá cho người đọc về sự việc này.

\section{2. Đa nguồn hạn định và đa nguồn mở rộng}

Trong ngữ liệu phân tích, cả hai loại đa nguồn đều xuất hiện với tần suất khác nhau. Đa nguồn hạn định xuất hiện ít hơn so với đa nguồn mở rộng $(35,4 \%$ so với $64,6 \%)$. Điều này cho thấy các tác giả bài bình luận có vẻ không chắc chắn lắm về đề tài này, và vì vậy họ luôn có xu hướng thỏa hiệp và khuyến khích người đọc tự chọn cho mình một thái độ đối với vụ việc.

Các yếu tố ngôn ngữ được các bài bình luận sử dụng để tạo sự tương tác với độc giả được thể hiện rõ ràng ở bảng sau:

\begin{tabular}{|l|c|c|c|}
\hline Yếu tố ngôn ngữ & $\begin{array}{c}\text { Đa nguồn } \\
\text { mở rộng }\end{array}$ & $\begin{array}{c}\text { Đa nguồn } \\
\text { hạn định }\end{array}$ & Tổng \\
\hline $\begin{array}{l}\text { Phụ ngữ tình thái } \\
\text { (có lẽ, có vé, chăc } \\
\text { chắn...) }\end{array}$ & $17,4 \%$ & $8,8 \%$ & $26,2 \%$ \\
\hline $\begin{array}{l}\text { Động từ trích dẫn } \\
\text { (nói, cho biết, } \\
\text { khằng dịnh...) }\end{array}$ & $20,7 \%$ & $12,6 \%$ & $33,3 \%$ \\
\hline $\begin{array}{l}\text { Từ tương phản và } \\
\text { phủ định (nhung, } \\
\text { mặc dù... nhung, } \\
\text { tuy vậy, chura bao } \\
\text { giờ...) }\end{array}$ & $0 \%$ & $7,6 \%$ & $7,6 \%$ \\
\hline $\begin{array}{l}\text { Động từ tình thái } \\
\text { (có thể, cần, phải, } \\
\check{a ́ t ~ l a ̀ . . .) ~}\end{array}$ & $15,1 \%$ & $6,4 \%$ & $21,5 \%$ \\
\hline $\begin{array}{l}\text { Cấu trúc phóng } \\
\text { chiếu (Theo Sky } \\
\text { News....) }\end{array}$ & $11,4 \%$ & $0 \%$ & $11,4 \%$ \\
\hline \multicolumn{1}{|c|}{ Tổng } & $64,6 \%$ & $35,4 \%$ & $100 \%$ \\
\hline
\end{tabular}

Có thể thấy rằng việc lựa chọn ngôn ngữ để thể hiện vị thế và sự tương tác với độc giả trong các bài bình luận của ngữ liệu là đa dạng, trong đó đa nguồn sử dụng yếu tố trích dẫn chiếm tỉ lệ khá cao trong các bài bình luận. Yếu tố này được thể hiện ở động từ trích dẫn (20.7\% đa nguồn mở rộng và $12,6 \%$ đa nguồn hạn định) và cấu trúc phóng chiếu $(13,4 \%)$. Các phụ ngữ tình thái và động từ tình thái cũng góp phần giúp các nhà bình luận hoặc tăng sự tin cậy của bài viết hoặc gia tăng sự thỏa hiệp với người đọc.

Trong dữ liệu có khá nhiều yếu tố tham thoại sử dụng yếu tố liên kết văn bản như: nhưng, dù vậy, dù...nhung. Martin \& White (2005) đặt tên loại tham thoại này là đa nguồn hạn định tương phản.

C23V16: Dù Noriega sau này bị Mỹ lật đổ nhung mối quan hệ của ông với các băng đảng đã góp phần khiến Panama nổi lên trở thành một thiên đường rưa tiền cho tọi phạm. (Hạn định tuoong phản)

Ngoài đa nguồn hạn định tương phản, các yếu tố ngôn ngữ khác của đa nguồn hạn định như các phụ ngữ tình thái chắc chắn, thực chất là, sự thực là, hay các động từ diễn tả quá trình hành vi hoặc phát ngôn như chứng thực rằng, xác nhận rằng, khẳng định, kết luận rằng, công bố.... Người viết thường sử dụng những yếu tố tham thoại này khi muốn thỏa hiệp với người đọc rằng những thông tin kèm theo những lời đánh giá này hoàn toàn chân thực, hợp lý, đáng tin cậy và tác giả rất muốn người đọc tin theo những gì được viết ra.

C8V23: ...và khi biết được rằng nhiều người giàu có đang lợi dụng các kỹ thuật né thuế hợp pháp để trục lợi, chắc chắn người dân sẽ không để yên. (Hạn định-khăng định)

C17V15: Các hoạt động rưa tiền thực chất là hơp thức hóa nhũng khoản tiền phi pháp. (Hạn định - khẳng định)

C9V29: Tổng thống Panama Juan Carlos Varela trong khi đó khănng đinh ông sẽ không khoan dung với tội phạm tài chính. (Hạn định - khẳng định)

Đa nguồn mở rộng thường được tác giả bài báo dùng khi đánh giá các thông tin không 
chắc chắn. Việc sử dụng đa nguồn mở rộng bằng những phụ ngữ tình thái như lẽ $r a$, có vé, có lẽ, hay những động từ tình thái như nên, phải, cần, có thể, có khả năng là, hoặc những ngữ phóng chiếu (Projections) như theo nguồn tin ..., được biết rằng, ông A nói... sẽ giúp người viết tạo cho mình một vị trí an toàn trong trình bày thông tin nhằm tăng sự thỏa hiệp với người đọc. Người viết có thể một phần tách mình ra khỏi trách nhiệm với thông tin và khuyến khích người đọc hoặc bất kì ai khác tự chọn cho mình một sự đánh giá thông tin.

C25V21: Thông điệp mà ho đưa ra trong nhiều bài phát biểu là nhũng gì mà giới siêu giàu làm hiện nay không vi phạm pháp luạt, nhung lễ ra đó phải là nhũng hành vi trái luạt. (Đa nguồn mở rộng)

C12V16: Tù thông tin này, Telegraph cho rằng có vẻ ông Putin đang quản lý luợng tiền khổng lồ thông qua các công ty vỏ bọc nêu trên. (Đa nguồn mở rộng)

Bằng việc sử dụng từ "lẽ $r a$ " và "có vê" trích dẫn từ tờ báo Telegraph người viết bình luận hàm ý muốn nói chúng tôi không chắc chắn lắm về suy đoán của mình, và người đọc có quyền chọn cho mình một góc đánh giá khác. Khi sử dụng những từ loại này, người viết rõ ràng biết rằng nhiều độc giả có khả năng có một cái nhìn về vụ việc khác với mình và họ hoàn toàn tôn trọng người đọc ở đây.

Ngoài ra, người viết báo cũng sử dụng các yếu tố khác như các động từ tình thái hoặc các phóng chiếu làm đa nguồn mở rộng như

C14V26: Khi tham nhũng, một chinh tri gia çần rửa số tiền bẩn để không bị lộ. (Đa nguồn mở rộng)

C3V7: Theo Sky News, đây là lần đầu tiên ông Cameron xuất hiện trước các chính trị gia kể tù khi ông thì̀a nhận huởng lợ tù̀ việc bán cổ phần trong quỹ đầu tu nước ngoài Blairmore Holdings của cha minh. (Đa nguồn mở rộng)

C18V24: "Đây là một chính phủ và đây nên là một quốc gia tin tuoơng vào khát vọng và sư giàu có", ông nói. (Đa nguồn mở rộng)

Với cách cung cấp nguồn trích dẫn " Theo Sky News" hoặc "ông nói", tác giả bài bình luận đã hàm ý rằng đây chỉ là một cách đánh giá từ một nguồn, độc giả có quyền thể hiện sự đánh giá của mình về sự vụ. White (1998) và Martin \& Rose (2003) gọi đây là một dạng quan điểm của người tường thuật tin (reporter voice) và họ cho rằng bằng việc trích dẫn nguồn, người viết có thể bày tỏ cảm xúc, thái độ của mình một cách hàm ẩn và an toàn.

Nhìn chung, sự xuất hiện đa nguồn ở các dạng khác nhau nhiều hơn so với đơn nguồn chứng tỏ người viết bình luận tin về "Hồ sơ Panama" luôn thể hiện quan điểm, thái độ với vụ việc, nhưng có vẻ như họ muốn tạo sự an toàn bằng việc sử dụng các nguồn ngôn liệu đánh giá từ các nguồn khác nhiều hơn từ chính bản thân. Và đây là không phải là một vụ việc xảy ra tại Việt Nam, nên người viết bình luận tỏ vẻ không chắc chắn về các nhận định của mình, vì vậy luôn tạo cơ hội cho người đọc thể hiện quan điểm bằng các đa nguồn mở rộng nhiều hơn các đa nguồn hạn định. Vì "Hồ sơ Panama" là một sự kiện chính trị chủ yếu ở nước ngoài nên các bài bình luận này mặc dù được ghi tác giả là người Việt nhưng vẫn có khả năng chỉ là một bài dịch từ tiếng Anh; tuy nhiên kết quả phân tích vẫn hoàn toàn phù hợp với những nghiên cứu trước đây của Vo (2001), Allison \& Wu (2005), Hyland (2005) và Lihua (2009) khi khẳng định yếu tố tham thoại thực sự hữu ích cho các nhà nghiên cứu 
trong việc tìm hiểu cách thức người viết thể hiện thái độ và cách họ giao tiếp với độc giả bằng các nguồn thái độ đó như thế nào.

\section{Kết luận}

Người viết bình luận tin "Hồ sơ Panama" luôn có xu hướng chọn các đa nguồn thay vì đơn nguồn trong các bài viết của mình. Các đơn nguồn xuất hiện rải rác nhằm giúp người viết truyền tải thông tin sự thực về vụ việc, còn các đa nguồn thì lại giúp người viết thể hiện mức độ chắc chắn của mình với thông tin trong bài viết, từ đó tương tác với người đọc và gây ảnh hưởng lên việc hình thành thái độ của người đọc với vụ việc. Việc sử dụng đa nguồn hạn định và đa nguồn mở rộng trong diễn ngôn giúp người viết báo thể hiện hoặc thế mạnh của mình trong việc thuyết phục độc giả tin vào những gì mình tin hoặc sự tôn trọng độc giả trong việc thỏa hiệp với độc giả về thái độ đối với vụ việc.

\section{Tài liệu tham khảo}

\section{Tiếng Việt}

Nguyen, T.T.H. (2016). Nghĩa liên nhân của ngôn ngữ trong thể loại bình luận báo chí tiếng Anh-nhìn từ Ngữ pháp Chức năng và Thuyết Đánh giá, Tù điển học \& Bách khoa thu, 3(41).109-114.

\section{Tiếng Anh}

Allison, D. \& Wu. S.M. (2005). Evaluative Expressions in Analytical Arguments: Aspects of Appraisal in Assigned English Language Essays. Journal of Applied Linguistics. 2(-1).105.

Halliday, M.A.K. (1994). An Introduction to Functional Grammar. London: Edvard Arnold.

Hyland, K. (2005). Stance and Engagement: a Model of Interaction in Academic Discourse, Discourse
Studies 7.173.

Lihua, L.. (2009). Discourse Construction of Social Power: Interpersonal Rhetoric in Editorials of the

China Daily. Discourse Studies 11(-1).

Martin, J. R. \& Rose, D. (2003). Working with Discourse. Meaning beyond the Claus. London:

Continuum.

Martin, J.R. \& White, P.R.R. (2005). The Language of Evaluation. Appraisal in English. Hampshire:

Palgrave Macmillan.

Tran,V.T.H. (2011). A Linguistic Study on Social Attitude towards the Quality Issues of Postgraduate

Education in Vietnam. Thesis of Doctor of Philosophy at Department of Education, University of Wollongong.

Vo, D.D. (2011). Style, Structure and Ideology in English and Vietnamese Business Hard News Reporting - A Comparative Study. Thesis of Doctor of Philosophy in Linguistics at Falcuty of Social Sciences and Humanities, University of Adelaide.

White, P.R.R. (2003). Beyond Modality and Hedging: A Dialogic View of the Language of Intersubjective Stance. Text - Special Edition on Appraisal.

White, P. R. R. (1998). Telling Media Tales: the News Story as Rhetoric. Ph.D. thesis, University of

Sydney. 


\title{
THE INTERACTION OF NEWSPAPER COMMENTARIES ON “THE PANAMA FILE" WITH POTENTIAL READERS: AN APPRAISAL ANALYSIS
}

\author{
Nguyen Thi Thu Hien \\ Department of Foreign Languages, Quy Nhon University \\ 170 An Duong Vuong, Quy Nhon, Binh Dinh, Vietnam
}

\begin{abstract}
The popularity of Appraisal Theory in discourse interpersonal meaning analysis has been increasing, especially in journalism discourses. Engagement is chosen as a tool to investigate the authors' stance and interaction with the potential readers of 30 commentaries on "The Panama file". The findings reveal that Heterogloss is used much more than Monogloss and that Expansion is more popular than Contraction. This result has proved a high level of communication between the writers and their imaginary readers. Moreover, the commentators have implicitly either made the readers follow their attitude by Contraction or given the readers chances to choose their own evaluation on the information by Expansion.
\end{abstract}

Keywords: Appraisal Theory, Engagement, Heterogloss, Monogloss, Expansion, Contraction 